Article

\title{
Cantilever-Droplet-Based Sensing of Magnetic Particle Concentrations in Liquids
}

\author{
Wilson Ombati Nyang'au 1,2,*(D), Andi Setiono ${ }^{1,3}$, Maik Bertke ${ }^{1}$, Harald Bosse ${ }^{4}$ and \\ Erwin Peiner ${ }^{1}$ D
}

1 Institute of Semiconductor Technology (IHT) and Laboratory of Emerging Nanometrology (LENA), Technische Universität Braunschweig, 38106 Braunschweig, Germany; a.setiono@tu-braunschweig.de (A.S.); m.bertke@tu-braunschweig.de (M.B.); e.peiner@tu-braunschweig.de (E.P.)

2 Department of Metrology, Kenya Bureau of Standards (KEBS), Nairobi 00200, Kenya

3 Research Center for Physics, Indonesian Institute of Sciences (LIPI), Kawasan Puspiptek Serpong, 15314 Tangerang Selatan, Indonesia

4 Precision Engineering Division, Physikalisch-Technische Bundesanstalt (PTB), 38116 Braunschweig, Germany; Harald.Bosse@ptb.de

* Correspondence: wilombat@tu-braunschweig.de

Received: 30 September 2019; Accepted: 31 October 2019; Published: 1 November 2019

\begin{abstract}
Cantilever-based sensors have attracted considerable attention in the recent past due to their enormous and endless potential and possibilities coupled with their dynamic and unprecedented sensitivity in sensing applications. In this paper, we present a technique that involves depositing and vaporizing (at ambient conditions) a particle-laden water droplet onto a defined sensing area on in-house fabricated and commercial-based silicon microcantilever sensors. This process entailed the optimization of dispensing pressure and time to generate and realize a small water droplet volume $\left(V_{\mathrm{d}}=49.7 \pm 1.9 \mathrm{pL}\right)$. Moreover, we monitored the water evaporation trends on the sensing surface and observed total evaporation time per droplet of $39.0 \pm 1.8 \mathrm{~s}$ against a theoretically determined value of about $37.14 \mathrm{~s}$. By using monodispersed particles in water, i.e., magnetic polystyrene particles (MPS) and polymethyl methacrylate (PMMA), and adsorbing them on a dynamic cantilever sensor, the mass and number of these particles were measured and determined comparatively using resonant frequency response measurements and SEM particle count analysis, respectively. As a result, we observed and reported monolayer particles assembled on the sensor with the lowest MPS particles count of about $19 \pm 2$.
\end{abstract}

Keywords: microcantilever; resonant frequency; magnetic polystyrene particles; droplet; adsorption; mass; concentration

\section{Introduction}

With the recent advancement in micro- and nanofabrication technologies, cantilever-based sensors have increasingly become versatile tools for various sensing applications due to their fascinating intrinsic flexibility and robustness. They have widely been embraced and applied in many scientific fields ranging from physical and chemical sensing, material characterization, magnetometry, explosive detection, to biological disease diagnosis [1-4]. For instance, they have immensely and significantly contributed to the biopharmaceutical development, manufacture and quality assurance, in which the titer of virus is related to the viral dosage and resultant performance of the vaccine [5]. Moreover, these sensors play a critical role in protein aggregation studies, differentiation and concentration measurement of sub-visible protein aggregates and silicone oil droplets in pre-filled syringe aggregation studies. In drug delivery [6,7], cantilever-based sensors facilitate the measurement of the concentration 
and particle size of the delivery vector, which often influence the biological response to the drug. Similarly, in disease diagnostics, they have been deployed in determining the concentration of specific exosomes and microvesicles [8], which may be indicative of the onset of a disease. They are also widely used in nanoparticle toxicology studies [2] to determine the concentration of particles within a biological or ecological environment, which essentially influences the biological response to the nanoparticles from a toxicity perspective, etc.

In the measurement of particle concentration, for instance, some various measurement technologies such as dynamic light scattering, nanoparticle tracking analysis, and resonant mass sensing can be deployed. These technologies provide particle concentration measurement based on particle fluorescence, particle size analysis and ensemble particle zeta potential measurement, and particle mass measurements, respectively. For the latter, differential stress at the surface to the beam of the cantilever causes bending or deflection of the cantilever. Most equipment for determining small masses, e.g., mass spectrometers [9] and suspended microchannel resonators [10], often utilize complicated technology and are quite expensive. On the other hand, the scope of application of Quartz Crystal Microbalances (QCMs) has been extended beyond gaseous to liquid media (e.g., in the study of the environment, nanoparticles, etc.). However, the interaction of the QCM surface with the liquid often results in the formation of soft or viscoelastic films. Besides, spurious factors (e.g., stress, pressure, etc.) exist [11], which potentially dampen the vibrations. This has subsequently necessitated the recent deployment of QCM with dissipation monitoring [12] to measure both the resonant-frequency change $(\Delta f)$ and energy loss (measured as dissipation change).

In providing a low-cost measurement approach, dipping a cantilever sensor into a magnetic particle solution has also recently been devised and implemented [13]. However, this particle sampling and measurement approach results in low quality factor $(Q \sim 180)$ due to severe damping and often yields randomized adsorption of particles on the whole cantilever surface. Furthermore, uniformity in particle adsorption or distribution over the sensing region is never guaranteed. In our study, we explored and presented an alternative droplet-based approach that can be utilized to precisely deposit particles on a specified sensing area on a cantilever for particle concentration or mass sensing applications. We successfully demonstrated localized deposition (utilizing liquid dispensing) of monodispersed magnetic polystyrene (MPS) particles onto a piezoresistive silicon cantilever sensors. Furthermore, we discussed some uncertainty parameters that influenced our measurements.

Through an optimized liquid-dispensing mechanism, we sub-sampled and loaded small water droplets with and without particles onto in-house fabricated cantilever sensors. Additionally, as a self-validating process, particle-laden droplets were similarly deposited on surface-treated silicon substrates and upon solvent evaporation, particle count estimations were performed using scanning electron microscopy (SEM). We also monitored and determined the droplet evaporation time $\left(t_{\mathrm{ev}}\right)$ and trends thereof for water droplets of known volume $\left(V_{\mathrm{d}}\right)$. The particles used herein included magnetic polystyrene (MPS) from Micromod $\mathrm{GmbH}$, and polymethylmethacrylate (PMMA) from Sigma-Aldrich Inc. It is worth noting that the two test particles have comparable (nominal) diameters and densities, but contrasting magnetic properties, with MPS and PMMA being magnetic and non-magnetic, respectively. However, similar to silicon, PMMA has magnetic susceptibility of less than 1 and is thus diamagnetic in nature. The essence of using these two particle types was to compare the results and assess the performance of our measurement approach.

Magnetic polymer particles (such as MPS) are usually synthesized by embedding magnetic nanoparticles (e.g., from pure metals such as $\mathrm{Fe}$, $\mathrm{Co}$ and $\mathrm{Ni}$ or their oxides) into a non-magnetic spherical (polymer) matrix [14]. Magnetic beads come in different compositions, sizes and shapes. These parameters determine their chemical and physical properties. These particles can be coupled with ligands that target specific molecules. This consequently makes them highly versatile and allows their widespread use in industrial and biomedical applications. For instance, in biomedicine, they are often useful in diagnosis and therapy, e.g., sorting and purification of cells, proteins and nucleic acids, as contrast reagents in magnetic resonance imaging (MRI), targeted drug delivery, diagnostics and 
targeted destruction of tumors by intercellular hyperthermia [15-17]. In the industry, these particles similarly play a critical role, e.g., as inorganic solar cell constituents, chemically inert additives, cosmetic UV protection and color filters for LCDs [18], optimization of bioengineering processes especially in the oil industry [14], high-density data storage $[19,20]$, etc. In environmental monitoring, magnetic nanoparticles can also be used directly for pollutant removal [21], e.g., from contaminated water resources. They for instance facilitate the removal of mercury ions in aqueous solutions by sensing, sorting and quantifying them using a nanoparticle-based system with optical and electrochemical detection regimes [22].

\section{Materials and Methods}

\subsection{Magnetic Polystyrene Particles}

In this study, we used magnetic polystyrene (MPS) beads of nominal diameter $\sim 2 \mu \mathrm{m}$ and density $1.1 \mathrm{gcm}^{-3}$, from micromod Partikeltechnologie GmbH (D-18119 Rostock, Germany). They are basically monodispersed particles consisting of magnetite $\left(\mathrm{Fe}_{3} \mathrm{O}_{4}\right)$ around an organic matrix of a styrene-maleic acid (SMA) copolymer, which are finally encapsulated with a polymer (polystyrene) layer [14] Hence, MPS exhibits a superparamagnetic behavior. The surface of the particles, herein used, is not chemically functionalized.

Originally, monodispersed MPS particle solution was obtained in an aqueous suspension of $50 \mathrm{mg} / \mathrm{mL}$ concentration and thoroughly mixed (by sonication) prior to use or dilution. The solution was otherwise diluted with deionized water to tune the concentration levels, i.e., into approximately 20 , 10,1 and $0.1 \mathrm{mg} / \mathrm{mL}$. This was the main variable, besides the droplet size, through which the mass $\Delta m$ or the number $N_{\mathrm{p}}$ of particles was varied for every particle solution dispensed on the target substrate or sensing surfaces.

\subsection{Silicon Substrates Preparation and Surface Treatment}

The silicon bulk substrates $\left(\sim 15 \times 15 \mathrm{~mm}^{2}\right)$ used in this study were cut from an $n$-type $\mathrm{Si}(100)$ wafer (Siegert Wafer $\mathrm{GmbH}$, Aachen, Germany). These wafers had resistivity and thickness of 1-10 $\Omega \mathrm{cm}$ and $275 \pm 15 \mu \mathrm{m}$, respectively. After cleaving, the substrates were individually cleaned by: (a) boiling (5 min) in an oxidant solution containing a mixture of sulfuric acid $\left(\mathrm{H}_{2} \mathrm{SO}_{4}, 96 \%\right.$ ) and hydrogen peroxide $\left(\mathrm{H}_{2} \mathrm{O}_{2}, 30 \%\right)$ in a volume ratio of $1: 1$; (b) immersing in a water bath (for $5 \mathrm{~min}$ ); (c) rinsing with deionized water; and (d) blow drying with nitrogen gas. Such a cleaning process was necessary in removing organic contaminants (e.g., dust particles, grease, silica gel, etc.) and ionic or metal contaminants from the silicon surface, prior to use or surface treatment.

Upon cleaning, some pre-selected bulk substrates were then dipped in HF solution (6-7\%) for about $5 \mathrm{~s}$ (to etch the native oxide from silicon surface). Immediately thereafter, some set of samples were treated with $\mathrm{O}_{2}$ plasma (100-E, Technics Plasma $\mathrm{GmbH}$ ) for $2 \mathrm{~min}$ at RF power of $220 \mathrm{~W}$, while others were treated with PSS/PDDA/PSS [23]. This was meant to yield different wettabilities (i.e., surface properties). Consequently, water contact angle $\theta$ was measured for each set of substrates: as-cleaned and after $\mathrm{O}_{2}$ plasma treatment.

Measurement of contact angle was carried out by the sessile-drop method using a dispensing system integrated with an imaging system which comprises of a light source, a collimating mask, an adjustable stage, and a digital microscope camera (Mz-902, Oowl Tech Ltd., Hong Kong, China). The latter was helpful in visualizing and capturing the water droplet on each silicon substrate. Careful attention was taken to generate a homogeneous background behind the drop. For this reason, we positioned a diffuser between the lamp and the water droplet. The height of the camera was also adjusted so that the actual drop and its reflection on the silicon surface could clearly be observed. Consequently, the intersection point at the solid-liquid-air interfaces would precisely be determined. The internal nominal diameter of the dispensing tip (Nordson EFD Inc., East Providence, RI, USA) 
was $\sim 0.10 \mathrm{~mm}$, and a low pressure ( $\sim 0.06$ bar) was applied to dispense a droplet at room temperature $\left(21.8 \pm 0.5^{\circ} \mathrm{C}\right)$.

In determining the contact angle, the profile of the drop image was extracted by an image-fitting in Image [24] using the Low Bond Axisymmetric Drop Shape Analysis (LBADSA) method [25]. Primarily, by observing the shape of such a drop profile, the contact angle $\theta$ can be measured, in which case $\theta$ is the angle formed between the drop tangent and the solid surface at the tri-phase contact point of a simple spherical droplet, as depicted in Figure 1. This approach allows for calculation of $\theta$ from sessile drop images based on the Young-Laplacian equation (Equation (1)) for spherical approximation for half-angle method, in which $r_{\mathrm{c}}$ and $h$ denote the contact radius and the height of the drop above the solid surface, respectively.

$$
\frac{\theta}{2}=\tan ^{-1}\left(\frac{h}{r_{c}}\right)
$$

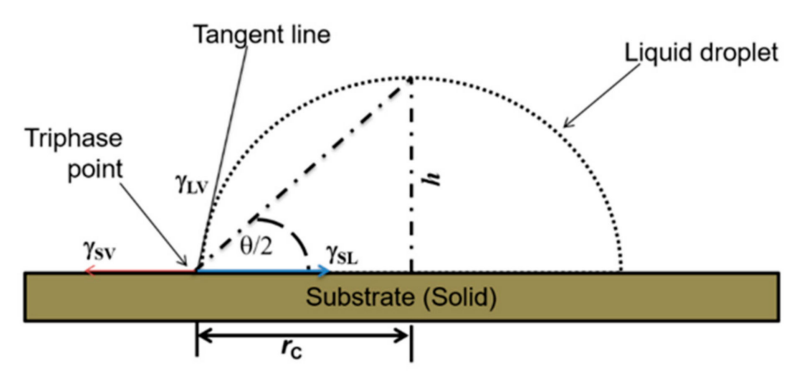

Figure 1. Schematic of contact angle $\theta$ measurement by the half-angle method.

Furthermore, the angle $\theta$ depends on the surface tensions for solid-liquid $\left(\gamma_{\mathrm{sl}}\right)$, solid-air $\left(\gamma_{\mathrm{sv}}\right)$, and liquid-air $\left(\gamma_{\mathrm{lv}}\right)$ interfaces. For small drops (of liquid density $\rho$ ), surface tension dominates over gravity, $g \approx 9.81 \mathrm{~ms}^{-2}$. Consequently, a drop tends to form a spherical-cap shape to minimize total free surface energy thereby resulting into a capillary length, or capillary constant $L_{\mathrm{c}}$, given as:

$$
L_{c}=\frac{\Delta \rho \cdot g}{\gamma_{l v}} .
$$

The parameter $\Delta \rho$ represents the difference in density of the involved phases (i.e., liquid and air). For water $\left(\rho=\rho_{\mathrm{w}}=1 \mathrm{gcm}^{-3}, \gamma_{\mathrm{lv}}=0.0728 \mathrm{Nm}^{-1}\right)$ at room temperature and standard pressure conditions, $L_{\mathrm{c}} \approx 1.35 \times 10^{-5} \mathrm{~m}^{-2}$. The LBADSA method further considers the pressure gradient across a surface to the drop curvature, and the contact angle is usually approximated based on a perturbation solution of the contour of an axisymmetric sessile drop and assumes gravity as the only external force, and follows Equations (2) and (3). Equation (3) relates the principal radii of curvature $\left(R_{1}\right.$ and $\left.R_{2}\right)$ at any point on the drop, the height $h$ of the drop, the capillary constant $L_{\mathrm{c}}$ and the radius of curvature $b$ at the apex.

$$
h=\frac{1}{L_{c}}\left(\frac{1}{R_{1}}+\frac{1}{R_{2}}-\frac{2}{b}\right)
$$

In our study, as-cleaned samples yielded $\theta=47.3^{\circ} \pm 2.7^{\circ}$, whereas contact angle after $\mathrm{O}_{2}$ plasma treatment $(2 \mathrm{~min})$ was about $9^{\circ}$. Obviously, our surface treatment with $\mathrm{O}_{2}$ plasma yielded enhanced hydrophilicity compared to the $24.5-46.5^{\circ}$ (for 1-20 min $\mathrm{O}_{2}$-RIE plasma treatment duration) reported by Alam et al. [26]. It is worth noting that a short-time lapse between surface treatment and contact angle measurements was observed in our study. Such short time gap does not compromise the results, which can otherwise be a consequence of buildup of static charges. Moreover, for our evaluation of the experimental contact angle, we assumed a negligible effect of gravity and a constant droplet volume. This can therefore be attributed to the measurement of contact angles immediately after $\mathrm{O}_{2}$ plasma treatment. 


\subsection{Fabrication of Silicon-Based Piezoresistive Cantilever Mass Sensors}

Fabrication process of our cantilever-based sensors was exclusively done in-house, using $n$-type (100) silicon (as a base material) and conventional bulk micromachining technologies as described by Wasisto et al. [27]. The process mainly entails substrate preparation (i.e., dicing the bulk Si wafer into $30 \times 30 \mathrm{~mm}^{2}$ pieces and cleaning them accordingly; cf. Section 2.2), thermal oxidation, photolithography, dopant diffusions (phosphorous and boron), etching processes (i.e., HF dip and cryogenic ICP-RIE: $\mathrm{SF}_{6} / \mathrm{O}_{2}$ ), contact holes formation, and metallization of electrical contacts $(\mathrm{Cr} / \mathrm{Au}=300 / 3000 \AA)$ coupled with a lift-off process. The general cantilever fabrication process utilizing bulk micromachining is schematically illustrated in Figure 2.

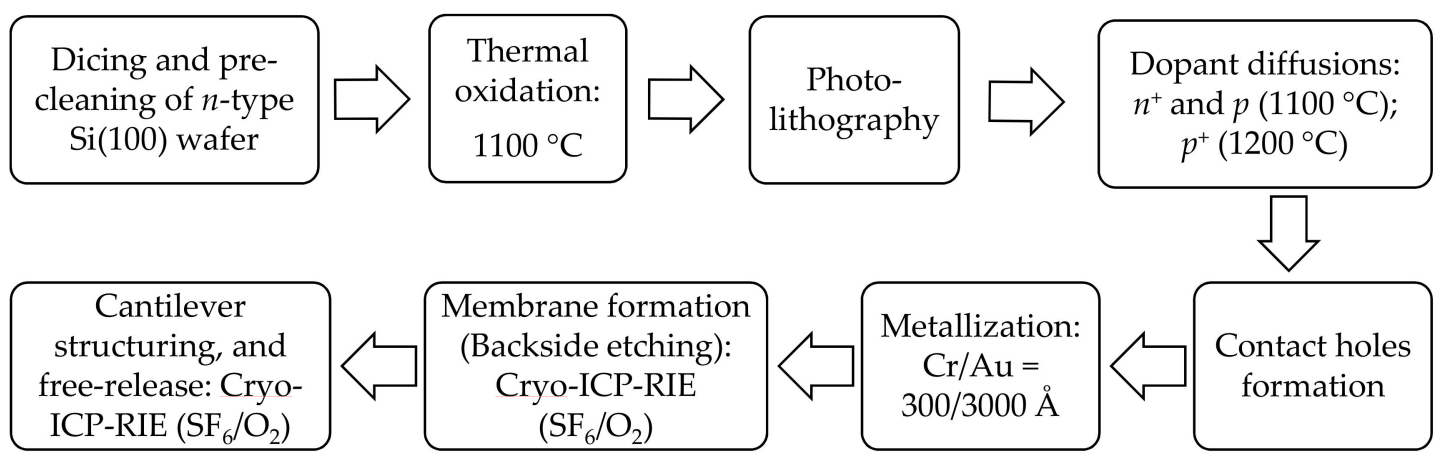

Figure 2. Schematic illustration of the main parameters and processes undertaken during the in-house fabrication of silicon-based cantilever sensors by bulk micromachining.

Fabrication process basically began with the deposition of an isolation layer $\left(\mathrm{SiO}_{2}\right)$ on top of the pre-cleaned silicon substrate at $1100{ }^{\circ} \mathrm{C}$ using thermal oxidation process. Hereafter, an intermediary substrate-cleaning process was performed by sonication in acetone and water rinses followed by nitrogen blow-dry. The silica layer $(\sim 100 \mathrm{~nm})$ provided an excellent protection during dopant diffusion processes, in which case heating furnace temperature was set and maintained at $1100{ }^{\circ} \mathrm{C}\left(n^{+}\right.$- and $p$-diffusions) and at $1200^{\circ} \mathrm{C}\left(p^{+}\right.$-diffusion).

Photolithography was then undertaken to pattern and transfer various geometric shapes/structures (e.g., U-shaped Wheatstone bridge piezo resistors, connection lines and contact pads) on a mask to the surface of the silicon substrates. A preliminary surface preparation process involving bubbling of hexamethyldisilazane (HMDS) within an enclosed chamber was executed (at $\sim 115^{\circ} \mathrm{C}$ for $\sim 5 \mathrm{~min}$ ) to enhance hydrophobicity of the substrate. Hereafter, pattern transfer ensued. Firstly, the prepared substrate was held onto a vacuum chunk and a photoresist (AZ 5214) was dispensed onto the silicon surface and spin-coated (e.g., $3500 \mathrm{rpm}$ for $35 \mathrm{~s}$ ). The sample was then partially evaporated in a soft baking process $\left(110^{\circ} \mathrm{C}\right.$ for $\left.\sim 50 \mathrm{~s}\right)$ to improve adhesion and uniformity, and optimize light absorbance characteristics of the photoresist. To transfer the mask image to the resist-coated wafer, both the mask and substrate were mounted onto respective stages/holders on the mask aligner equipment (MBJ4, SÜSS MicroTec AG, Garching, Germany) followed by alignment and UV exposure (for specified time period, e.g., $35 \mathrm{~s}$ ). To dissolve the soluble areas of the exposed photoresist and realize visible patterns on the wafer, the sample was immersed and gently agitated in a developer chemical solution (AZ 726) for $\sim 60 \mathrm{~s}$ and thoroughly rinsed with deionized water. The resulting pattern after resist development would then be inspected (under a light microscope) followed by a hard-baking process (at $100{ }^{\circ} \mathrm{C}$ for $\sim 30 \mathrm{~min}$ ). Subsequently, the hardened-patterned sample was immersed in a buffered hydrofluoric acid for about $10 \mathrm{~min}$ to remove the entire $\mathrm{SiO}_{2}$ layer or $\sim 5 \mathrm{~s}$ to clear the native oxide as desirable.

After forming piezo resistors, contact holes, electrical connections and contact pads, the sample was cryogenically etched (ICP-RIE, with $\mathrm{SF}_{6} / \mathrm{O}_{2}$ ) from the backside to create a membrane. Lastly, the patterning process (by lithography) was undertaken to form the desired free-ends (i.e., rectangular 
or triangular beam configurations [28]) of our cantilever sensors. This was then followed by the free-releasing of the cantilever sensors from the sample through a cryogenic dry etching process. The length and width of rectangular cantilevers were $1000 \mu \mathrm{m}$ and $170 \mu \mathrm{m}$, respectively, while the size of the equilateral-shaped free-end triangular cantilever was $\sim 700 \mu \mathrm{m}$. The thickness of our sensors was approximately $37.9 \pm 0.2 \mu \mathrm{m}$, and the mass $m_{0}$ of cantilever was $15.01 \pm 0.11 \mu \mathrm{g}$ and $24.65 \pm 0.18 \mu \mathrm{g}$ for rectangular and triangular cantilevers, respectively.

\subsection{Droplet Dispensing Apparatus}

Water droplet with/without MPS particles was generated from a needle tip (of specified size) by applying specified dispensing pressure over a defined pulse duration. By keeping the size of the needle tip constant, the drop size could reliably be tuned mainly by varying the magnitude of the dispensing pressure and/or time. Through X-ray computer tomography (xCT) measurements (undertaken at PTB), we precisely calibrated and determined the internal and external diameters of our dispensing stainless needle tip (see Figure 3) to be $0.1169 \pm 0.004 \mathrm{~mm}$ and $0.2357 \pm 0.0005 \mathrm{~mm}$, respectively. The latter was also necessary and helpful in calibrating our USB digital camera (Mz-902, Oowltech).

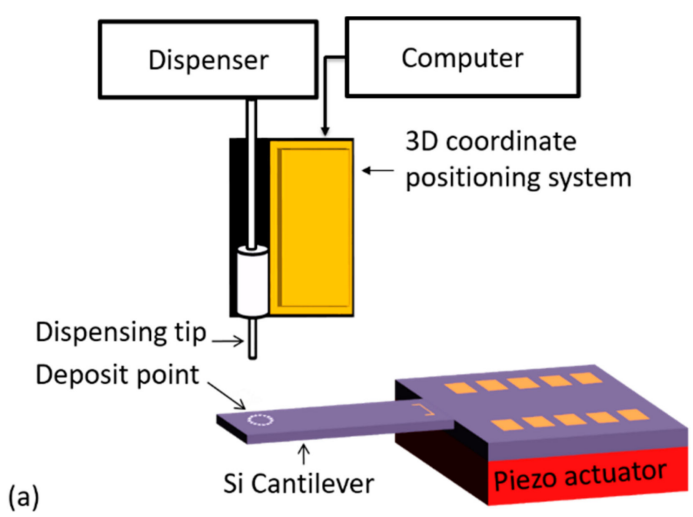

Figure 3. (a) Schematics of liquid (and particles) dispensing setup; and (b) X-ray computer tomography $(\mathrm{xCT})$ image of the dispensing stainless needle tip.

Firstly, each cantilever mass sensor was mounted on top of a piezo actuator within the sampler and carefully aligned in the horizontal plane. At this point, the particles collection target point would be defined $(X, Y)$, i.e., by specifying a coordinate along the axisymmetric and the vibrating axis of the cantilever. The needle tip would then be mounted into a barrel (containing a thoroughly mixed solution of MPS particles) and aligned directly on top of deposit target point. During the alignment process, a 3D micro-positioning system (with a resolution of $10 \mu \mathrm{m}$ ) was used to carefully move the needle tip to the target point; and with an aid of a camera, the height from the sensor and the tip would carefully be adjusted (Z-coordinate). To further enhance the accuracy of positioning the tip on the sensing surface and minimize sensor breakages, a camera was also incorporated in the alignment process. The tip was aligned and adjusted very closely above the sensor target deposit point, in orders of few microns. Finally, the droplet was generated (1500XL, Nordson EFD Inc., Rhode Island,USA) and deposited on the sensor by applying defined dispensing parameters (i.e., air pressure and pulse duration). Typically, minimum dispensing pressure ( $\sim 60 \mathrm{mbar})$ was applied and different pulse durations were used. This was desirable in generating a small droplet as much as possible.

\subsection{Resonant Mass Sensing Instrumentation}

In detecting cantilever bending due to the mass of the droplet or adsorbed particles, different sensing or read-out methods can be deployed, such as piezoelectric, electrodynamic, tunneling, hard contact, optical reflection, interferometric, capacitive, and piezoresistive techniques [29]. The latter mechanism was preferably integrated into our cantilevers during the fabrication process and was 
realized in the form of a U-shaped Wheatstone bridge by diffusing piezo resistors into the cantilever. To supply voltage $\left(1 \mathrm{~V}_{\mathrm{dc}}\right)$ and detect the voltage output from the bridge, a lock-in amplifier (MFLI, Zurich Instruments Ltd.) was connected to the cantilever contact pads (as shown in Figure 4). Since our microcantilevers were externally excited with an in-plane piezoelectric stack actuator (P-121.05, from PI Ceramics $\mathrm{GmbH}$, Lederhose, Germany), an excitation voltage (9.9 $\mathrm{V}_{\mathrm{pp}}$ ) was similarly supplied to the actuator by the MFLI instrument. The connections to and from the MFLI were accomplished through coaxial cables and SubMiniature version A (SMA) connectors. The required MFLI parameters (e.g., input voltage) and the signal read-out from sensor (e.g., frequency response) were managed using a computer via a control software.

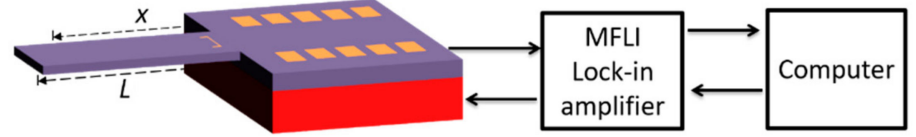

(a)

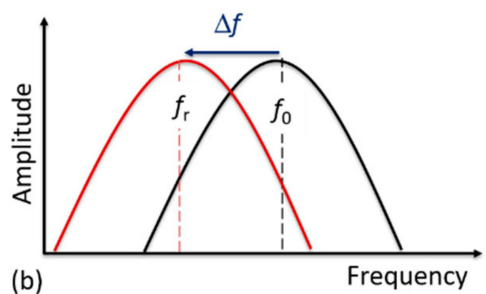

(b)

Frequency

Figure 4. Schematic illustration depicting: (a) the resonant frequency measurement setup; and (b) the expected resonant frequency shift $\Delta f$ due to the mass $\Delta m$ of attached media.

Initially, and for each particle adsorption cycle, the resonant frequencies of the cantilever before $\left(f_{0}\right)$ and after $\left(f_{\mathrm{r}}\right)$ load deposition were measured. This was necessary to precisely determine the resonant frequency shift $\left(\Delta f=f_{\mathrm{r}}-f_{0}\right)$ due to the loaded mass $\Delta m$ (i.e., mass of the droplet or adsorbed particles). Consequently, the particle and/or droplet mass was determined at a point $x$ from the fixed end of the cantilever beam of length $L$. With the measured resonant frequency shift $\Delta f$, the mass of the cantilever $m_{0}$ and the mode-shape function $U\left(x_{\Delta \mathrm{m}}\right)$, the drop or adsorbate mass $\Delta m$ can be calculated according to [30]:

$$
\Delta m=\frac{m_{0}}{U^{2}\left(x_{\Delta m}\right)}\left(\frac{f_{0}^{2}}{f_{r}{ }^{2}}-1\right),
$$

where $U\left(x_{\Delta \mathrm{m}}\right)=(\cos \lambda+\cosh \lambda)\left(\cos \left(\lambda \frac{x}{L}\right)-\cosh \left(\lambda \frac{x}{L}\right)\right)+(\sin \lambda-\sinh \lambda)\left(\sin \left(\lambda \frac{x}{L}\right)-\sinh \left(\lambda \frac{x}{L}\right)\right)$. The parameter $\lambda$ represents a modal constant, which is about 1.8751 for the first mode. To begin a new measurement cycle, the adsorbed particles were removed/cleaned from the cantilever surface and $f_{0}$ was measured again to average and compare with the initially obtained value.

\subsection{Cleaning and Removal of Adsorbed Particles from the Cantilever}

Whenever particles or analytes are collected on a silicon cantilever for mass detection or otherwise, they often adhere on the sensing surface. For instance, airborne particles such as cigarette smoke sticking on the surface of silicon cantilever can easily be removed by immersing and gentle agitating the cantilever in a glass containing acetone (for a few minutes) [31]. However, after adsorbing liquid-dispersed MPS particles on the silicon cantilever, conventionally much more effort is required to remove and clear these particles from the sensing surface.

In this work, therefore, regeneration of particle-adsorbed cantilever sensors (Figure 5a) was mainly performed by immersion in acetone and sonication for about 3-5 min. With this approach, the particles were detached and cleared from the surface (Figure 5b). Since cleaning the fragile silicon cantilevers by sonication is a risky process, often bound to break and destroy the sensors, we clipped each particle-laden cantilever on the flat surface of a metallic mounting before immersing it in a glass containing acetone. This mechanism was successfully implemented and resulted in nearly $100 \%$ particle removal efficiency and alleviated the risk of damaging the cantilevers. 
(a)

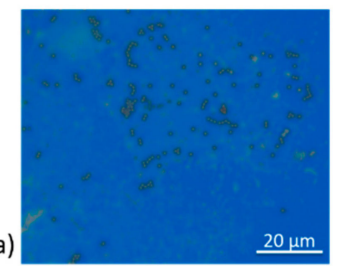

(b)

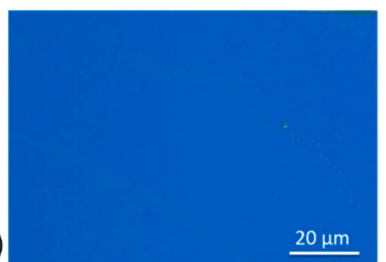

Figure 5. Optical images of a silicon cantilever surface: (a) containing adsorbed magnetic polystyrene beads (dark round features); and (b) after sonication treatment (in which all particles are completely removed from the surface).

\section{Droplet Size Optimization and Evaporation}

\subsection{Droplet-Size Measurements}

By varying dispensing time and output pressure, various droplet sizes would be obtained. It was clearly observed (Figure 6) that, for the same dispensing pressure, the size of the water droplet increased with the pulse duration $(t)$. By multiplying droplet mass with the water density $\left(\rho_{\mathrm{w}}=1 \mathrm{gcm}^{-3}\right)$, the drop volume was obtained. Obviously, a small droplet size $V_{\mathrm{d}}=49.7 \pm 1.9 \mathrm{pL}$ was measured for $t=10 \mathrm{~ms}$ for a dispensing pressure $p=60$ mbar. Similarly, $V_{\mathrm{d}}=98.1 \pm 1.0 \mathrm{pL}$ and $0.2 \mathrm{~nL}$ corresponded to $t=0.1 \mathrm{~s}$ for $p=0.06$ bar and 0.2 bar dispensing air pressures, respectively.

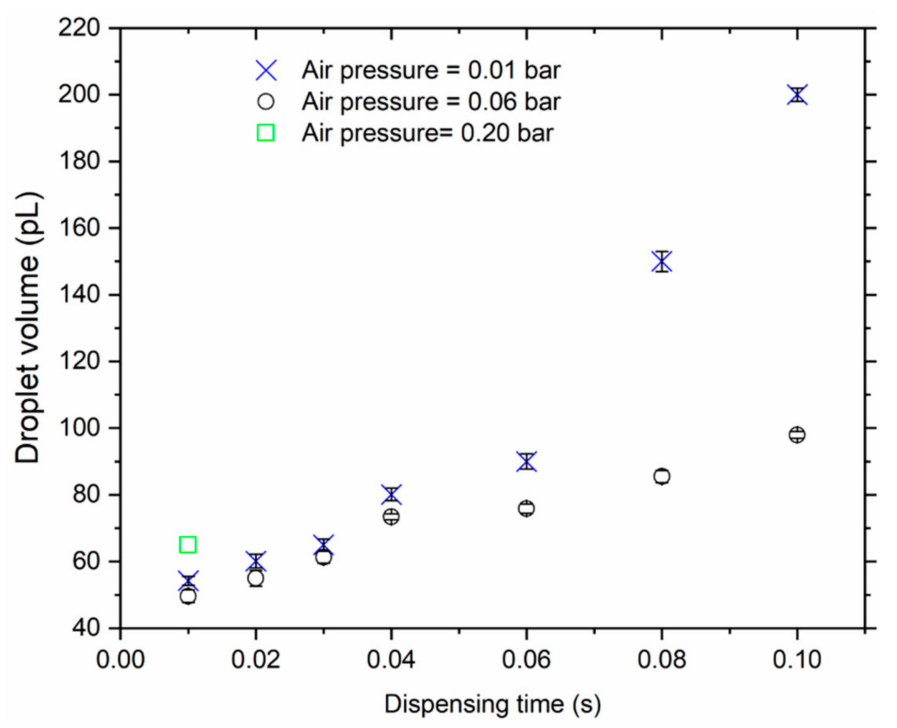

Figure 6. Volume of water droplets dispensed from a needle tip (of nominal internal diameter = $0.10 \mathrm{~mm}$ ) on a cantilever sensor under various dispensing air pressures (0.06-0.20 bar) and pulse durations (0.01-0.10 s) at ambient conditions $\left(T=23.3 \pm 0.5{ }^{\circ} \mathrm{C} ; r H=33 \pm 4 \%\right)$.

Consequently, we used low output air pressure (60 mbar) and the shortest pulse duration $(10 \mathrm{~ms})$ to generate and realize all the droplets particularly aimed at sampling the particles from the solution. The quality factor $Q$ before and after depositing the water droplet on the cantilever were, respectively, $1800 \pm 250$ and $153 \pm 50$. The latter shows a significant reduction in $Q$, which obviously depicts severe damping of the resonator when the water rests on the sensing surface. By assuming a spherical-shaped droplet of $V_{\mathrm{d}} \approx 50 \mathrm{pL}$, a diameter $\sim 45.6 \mu \mathrm{m}$ was computed, which is much smaller than the width of the cantilever sensor $(w \approx 170 \mu \mathrm{m})$.

\subsection{Evaporation Dynamics of Small Droplets on Microcantilever Surface}

Water microdroplets on a cantilever sensor are bound to evaporate at ambient conditions. In our study, the loss of water droplet mass from the sensing surface was monitored by measuring the 
shift in resonant frequencies over time (as delineated in Figure 7a). This was particularly necessary to determine the droplet evaporation time $t_{\mathrm{ev}}$, and the minimum particle adsorption or waiting time prior to particle-mass measurement. Therefore, in determining $t_{\mathrm{ev}}$, the loss of droplet mass was initially monitored over time under ambient conditions through an offline resonant frequency measurement system (using a MFLI instrument). It should be noted (Figure 7a) that upon depositing the water droplet on the cantilever sensor, an immediate shift in resonant frequency was observed (i.e., $f_{0} \sim 215.415 \mathrm{kHz}$ shifted to $f_{\mathrm{r}} \sim 213.968 \mathrm{kHz}$ ), which corresponds to the added mass by the droplet. The total time taken for the cantilever to completely lose water from the surface and restore $f_{\mathrm{r}}$ to almost $f_{0}$ (except for the shift induced by the mass of the attached particles) is equivalent to the drop evaporation time, $t_{\mathrm{ev}}=39.0 \pm 1.8 \mathrm{~s}$.
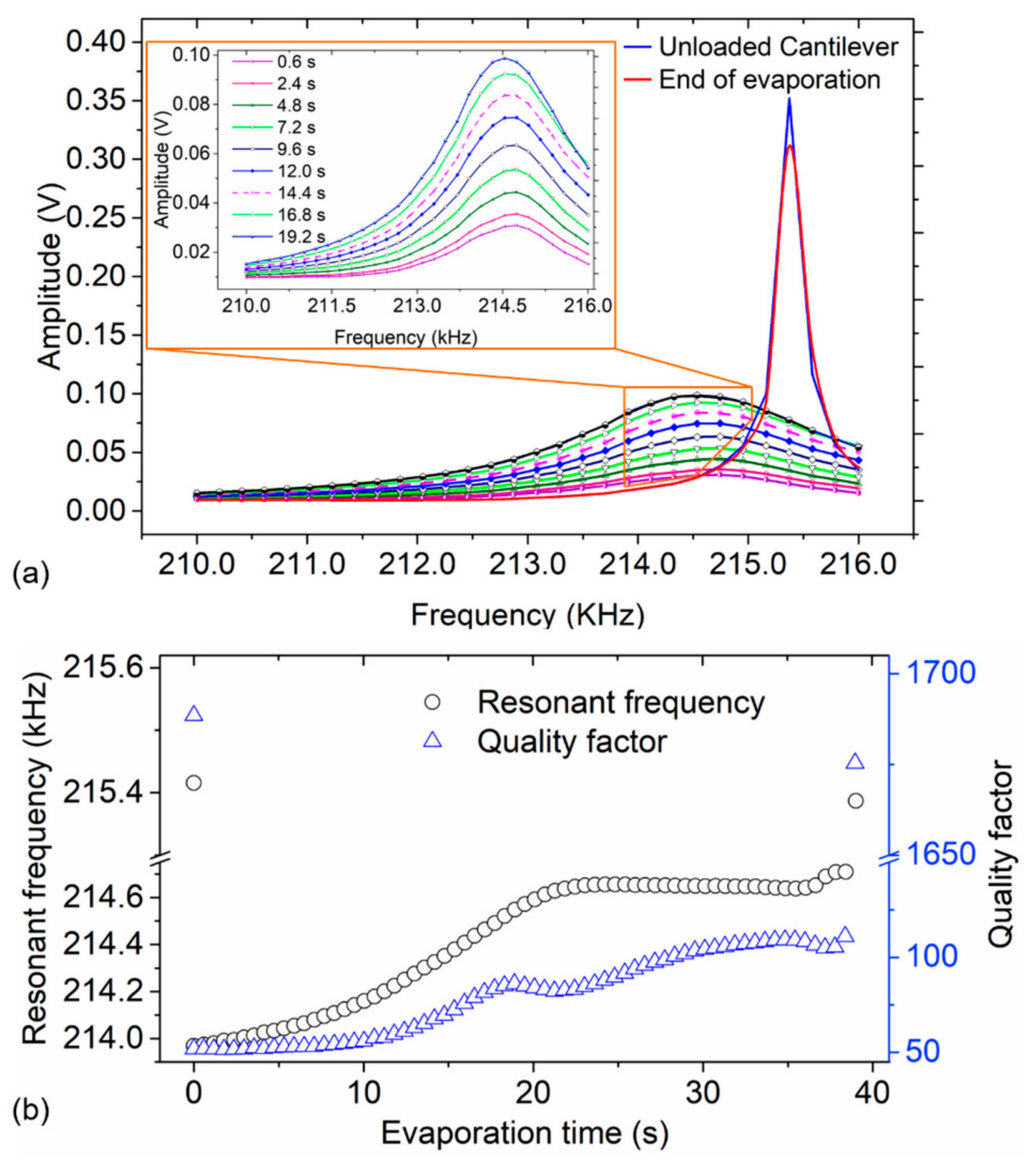

Figure 7. Offline monitoring of an evaporating water droplet $(\sim 50 \mathrm{pL})$ on a silicon-based piezoresistive microcantilever sensor under ambient atmospheric conditions $\left(T=24.4{ }^{\circ} \mathrm{C}, r H=36 \%\right)$. (a) Frequency responses before and after droplet deposition, and during droplet evaporation at different time intervals. (b) Resonance frequency $f_{0}$ and quality factor $Q$ determined by fitting of a single harmonic oscillator (SHO) model to the curves in (a) over time during the evaporation period for a $\sim 50 \mathrm{pL}$ water droplet.

It was clearly observed (in Figure 7a) that, once the water droplet was loaded onto the cantilever, the resonance frequency $f_{0}$ rapidly shifts to a minimum whereupon the sensor momentarily stabilizes and recovers from this droplet-deposit impact. The cantilever then loses the water $(\sim 50 \mathrm{pL})$ into the ambient atmosphere within a span of about 39 s. Rapid evaporation and instantaneously loss of the water towards the last evaporation period was however observed. Subsequently, this culminates to a rapid shift of frequency (i.e., back to $f_{0}$ ) and an abrupt rise in $Q$ (i.e., from $\sim 110$ to about 1700). The corresponding trends and changes in resonant frequency and quality factor were evidently found to significantly change with the evaporation time (as depicted in Figure 7b). In Figure 7, an instantaneous change (increase) in the evaporation rate towards the winding phase is evidently demonstrated. 
Contrarily, Arcamone et al. [32] observed a decrease in the evaporation rate with time during the evaporation process. Further work is therefore necessary to interrogate the cause of the sudden and rapid water loss from the sensing surface particularly towards the last phase of an evaporating water droplet.

To theoretically evaluate the approximate evaporation time, we consider a spherical droplet cap of height $h$, contact radius $r_{\mathrm{c}}$, radius of the sphere forming the spherical cap $R_{\mathrm{S}}$, and contact angle $\theta$ (in radians), having a volume $V_{\mathrm{c}}$ given by [33]:

$$
V_{\mathrm{c}}=\frac{\beta \pi}{3} R_{\mathrm{s}}^{3}
$$

where, $\beta=2-3 \cos \theta+\cos ^{3} \theta$ and $R_{s}=r_{c} / \sin \theta$. The relation of height of the spherical cap above the solid surface with $r_{\mathrm{c}}$ and $\theta$ is explicitly given in Equation (1) and can equivalently be related with $R_{\mathrm{S}}$ as: $h=R_{s}(1-\cos \theta)$. During droplet evaporation, the volume of the droplet on the solid surface at any time $t$ can be computed as follows [33]:

$$
V_{\mathrm{c}}^{2 / 3}=\left(V_{\mathrm{c}, \mathrm{i}}^{2 / 3}-\frac{2}{3} K \mathrm{f}(\theta) \cdot \mathrm{t}\right)(3 / 2),
$$

where $V_{\mathrm{c}, \mathrm{i}}$ denotes the initial droplet volume (at time $t=0 \mathrm{~s}$ ) and the term $2 / 3 K f(\theta) t$ represents the decrement in volume with time. The function $\mathrm{f}(\theta)=-\cos \left(\frac{\theta}{2 \ln (1-\cos \theta)}\right)$ is for the contact angle. For a constant contact-angle evaporation mode, we have $\mathrm{f}(\theta)=\mathrm{f}\left(\theta_{0}\right)$, where $\theta_{0}$ is the contact angle prior to evaporation $\left(\theta_{0} \sim 40^{\circ}\right.$ and $r_{\mathrm{C}}=0.044 \mathrm{~mm}$, i. e., $\mathrm{f}\left(\theta_{0}\right)=0.263676, \beta=0.1514, R_{\mathrm{S}}=0.070 \mathrm{~mm}$ and $\left.V_{\mathrm{c}, \mathrm{i}}=49.7 \mathrm{pL}\right)$. The parameter $K \approx 12.37467091 D \Delta c /\left(\rho_{\mathrm{L}} \beta^{1 / 3}\right)$, in which the symbols $D, \Delta c$ and $\rho_{\mathrm{L}}$ denote the diffusivity (in $\mathrm{cm}^{2} \mathrm{~s}^{-1}$ ) of the vapor molecules in the gas (i.e., air), the difference $c_{\mathrm{s}}-c_{\infty}$ between the saturation concentration of water vapor at the sphere surface $c_{\mathrm{S}}\left(\right.$ in $\left.\mathrm{gcm}^{-3}\right)$ and the ambient vapor concentration at infinite distance $c_{\infty}\left(\right.$ in $\mathrm{gcm}^{-3}$ ) to the droplet, and the liquid density (in $\mathrm{gcm}^{-3}$ ), respectively. Assuming complete loss of water from the sensing surface, Equation (6) thus reduces to:

$$
\mathrm{t}_{\mathrm{ev}}=\frac{3 V_{\mathrm{c}, \mathrm{i}}^{2 / 3}}{2 K \mathrm{f}\left(\theta_{0}\right)} .
$$

For a water droplet, $\rho_{\mathrm{l}}=\rho_{\mathrm{W}} \approx 1 \mathrm{gcm}^{-3}, D \approx 0.282 \mathrm{~cm}^{2} \mathrm{~s}^{-1}$ and $c_{\mathrm{s}}=p_{\mathrm{v}} M_{\mathrm{W}} /(R T) \sim 1.7081 \times 10^{-4} \mathrm{gcm}^{-3}$ given a molecular mass $M_{w}=18.01528 \mathrm{gmol}^{-1}$, the gas constant $R=8.314 \mathrm{~J}(\mathrm{~mol} \cdot \mathrm{K})^{-1}$ and the vapor pressure $p_{\mathrm{V}}$ in air of $22.2 \mathrm{mmHg}\left(\cong 29.6 \mathrm{mbar}\right.$ ) at a temperature $T \approx 24{ }^{\circ} \mathrm{C}[34] \mathrm{cm}^{2} \mathrm{~s}^{-1}$. Based on Equation (7), $V_{\mathrm{c}, \mathrm{i}}=49.7 \mathrm{pL}$ and $\mathrm{f}\left(\theta_{0}\right)=0.263676$, we can therefore estimate the total time taken for the water droplet to completely vanish from the surface of a cantilever sensor to be about $37.14 \mathrm{~s}$, which agrees very well with the experimental droplet evaporation time $\left(t_{\mathrm{ev}} \approx 39 \mathrm{~s}\right)$.

\section{Modeling Droplet-Particle Concentration: Wet and Dry Mass Approaches}

\subsection{Particle Adsorption on Cantilever and Resonant Frequency Responses}

Wet mass and dry mass of the droplet can be measured immediately after depositing and evaporating the droplet, respectively. In both cases, a shift in resonant frequency is observed. Firstly, in the case of a wet drop, a shift in the resonant frequency can be observed from $f_{0}$ (i.e., for a bare cantilever) to $f_{\mathrm{r} 1}$ (cantilever + water + particles) as the droplet lands on the cantilever. Secondly, after a time lapse of $t_{\mathrm{ev}}$ the water is expected to evaporate and completely vanish from the sensing surface thereby shifting the resonant frequency $f_{\mathrm{r} 1}$ to $f_{\mathrm{r} 2}$ (i.e., cantilever + particles). The mass of the adsorbed particles can therefore most conveniently be computed (using Equation (4)) from the frequency shift of $f_{\mathrm{r} 1}$ to $f_{\mathrm{r} 2}$, i.e., $\Delta f=f_{\mathrm{r} 2}-f_{\mathrm{r} 1}$. Similarly, the mass of the particle carrier fluid (i.e., water) can at the same time be determined from the frequency changes of $f_{\mathrm{r} 1}$ (wet mass) to $f_{\mathrm{r} 2}$ (dry mass), which nearly 
corresponds to the previously discussed evaporation trends (in Figure 7). In this study, however, since the evaporation time and droplet size had been determined previously, only $f_{\mathrm{r} 2}$ and $f_{0}$ were of essence.

A typical water droplet containing MPS particles is presented in Figure 8a, from which the wet mass can most conveniently be obtained. As shown in Figure $8 \mathrm{~b}$, particles were adsorbed on the sensing surface after water evaporation thereby making it possible to determine the dry mass. In Figure $8 c, d$, for instance, we present typical frequency responses of microcantilevers with and without adsorbed magnetic particles. From these responses, the mass of the MPS beads was correspondingly determined from the observed frequency shift $\Delta f \approx-4.77 \mathrm{~Hz}$ in accordance with Equation (1). These MPS beads were positioned at a point $x \approx 618.32 \pm 44.20 \mu \mathrm{m}$ (i.e., $U\left(x_{\Delta \mathrm{m}}\right) \approx-2.80$ ) from the fixed end of the cantilever. It should be noted that, for $x \approx L=1000 \mu \mathrm{m}$, the value of $U\left(x_{\Delta \mathrm{m}}\right) \approx-6.08$. The measurements shown Figure $8 \mathrm{c}$ were achieved with $Q=2080 \pm 430$, for both the bare and loaded-state of the cantilever.

(a)

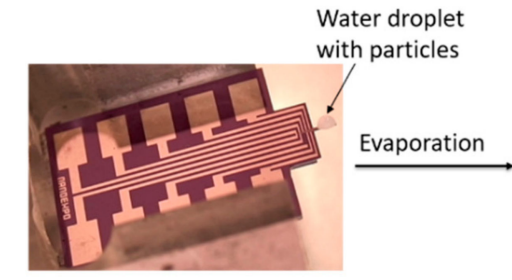

(b)

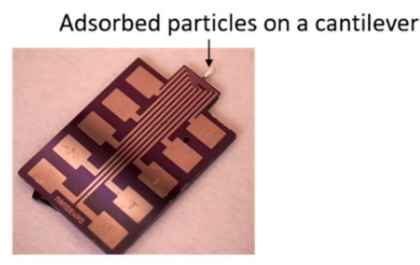

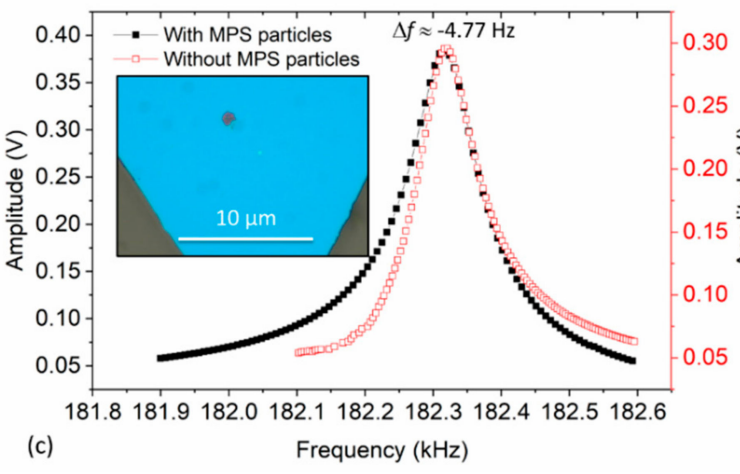

(c)

$$
\text { Frequency }(\mathrm{kHz})
$$

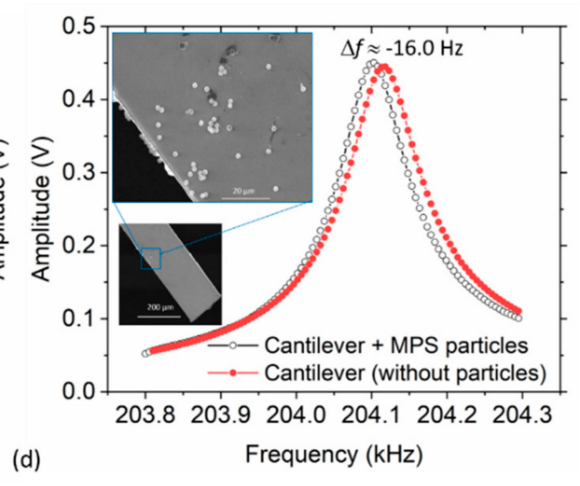

Figure 8. Optical images of: (a) particle-laden droplet on a cantilever, and (b) cantilever with adsorbed particles (after solvent evaporation). (c,d) Plots of resonant frequency measurements of a triangular and rectangular (normal) cantilever sensors with/without magnetic polystyrene (MPS) particles.

For comparability with commercial sensors, we similarly implemented and deposited MPS particles on a CiS cantilever sensor (CAN50-2-5, CiS Forschungsinstitut für Mikrosensorik GmbH, Germany, https://www.cismst.de/en/loesungen/mikrotastspitzen/). This silicon-based sensor was first cleaned, wire bonded (Figure 9a) and aligned (for particle deposition, cf. Section 2.4). The typical frequency response prior to and after adsorbing MPS beads on CiS cantilever is vividly shown in Figure $9 \mathrm{~d}$. The particles were adsorbed at a point $x \approx 4.80 \mathrm{~mm}$ from the fixed end, which apparently shifted the resonant frequency $\left(f_{0}=1.11 \mathrm{kHz}\right)$ by $-15.42 \pm 0.04 \mathrm{~Hz}$. This yielded a calculated particles mass of about $0.13 \mu \mathrm{g} \pm 0.3 \mathrm{ng}$, which is equivalent to about $36,473 \pm 84$ particles. This number was computed from the ratio of the adsorbate mass to single particle mass (which was estimated from the measured particle diameter of $1.83 \pm 0.03 \mu \mathrm{m}$ for MPS, assuming all particles are spherical in shape). From frequency response measurements (shown in Figure 9d), a quality factor $Q$ of about $379 \pm 3$ was realized, depicting a fairly stable operating environment. This cantilever was externally excited in its fundamental out-of-plane mode with a piezo actuator, and the connections to and from the MFLI amplifier were done (as illustrated previously in Figure 4). 


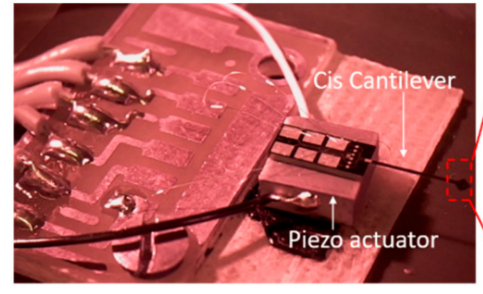

(a)

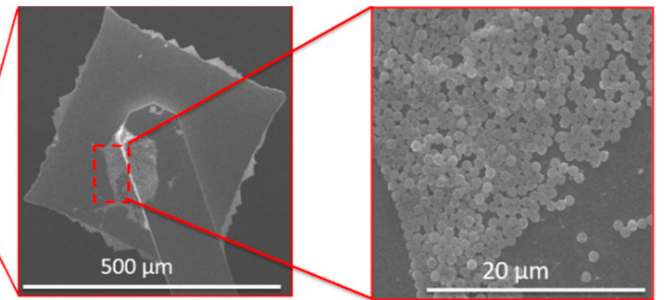

(c)

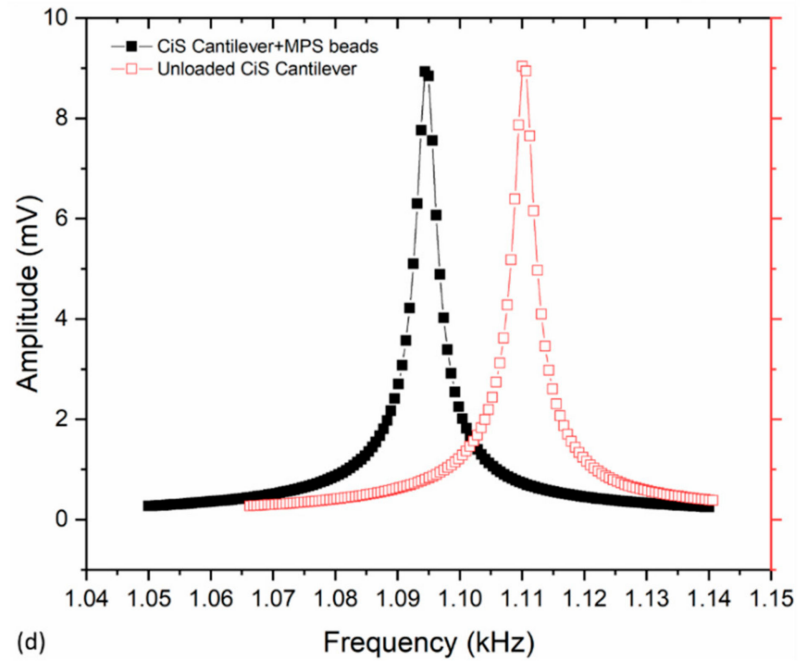

Figure 9. (a) Image of a wire-bonded CiS silicon cantilever sensor mounted on a thickness-extensional piezo-stack actuator; $(\mathbf{b}, \mathbf{c})$ SEM images of adsorbed MPS particles on the cantilever; and (d) measured resonance frequency responses before and after loading MPS beads on the sensor.

In Figure 10, we further present frequency responses due to adsorbed PMMA particles on a triangular cantilever (in-house) and a CiS cantilever (CAN30-1-2, $m_{0} \approx 20 \mu \mathrm{g}$, https://www.cismst. de/en/loesungen/mikrotastspitzen/). The observed resonant frequency shift in Figure 10a was about $-181.1 \mathrm{~Hz}$ (with $Q \approx 1700$ ) while in Figure $10 \mathrm{~b} \Delta f \approx-11.8 \mathrm{~Hz}$ (with $Q \approx 285$ ), corresponding to approximately $5.73 \mathrm{ng}$ (i.e., 1449 particles) and $4.07 \mathrm{ng}$ (i.e., 1036 particles), respectively. The number of calculated PMMA particles (based on point-mass condition) from other measurements ranged from about 56 to 6169 . The assumed particle density and measured diameter were $1.18 \mathrm{gcm}^{-3}$ and $1.90 \pm 0.05 \mu \mathrm{m}$, respectively. A comparison of the number of particles estimates (both for MPS and PMMA) based on resonant frequency responses and particle counts (from SEM) is presented in the next section.
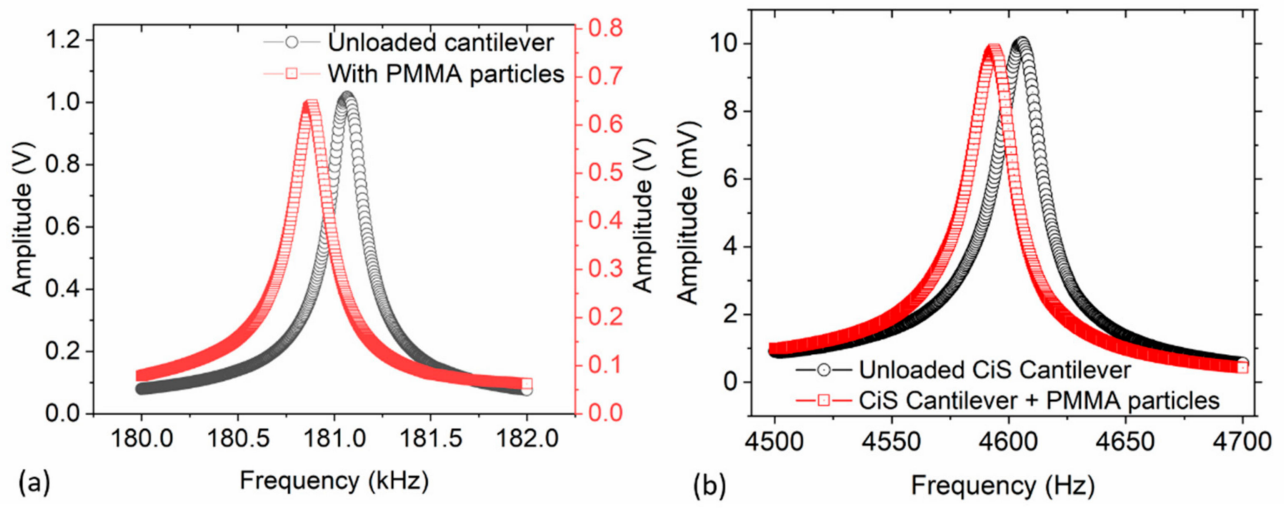

Figure 10. Frequency measurements depicting responses with and without PMMA particles using: (a) a triangular (in-house) cantilever; and (b) CiS commercial sensor. 


\subsection{Microscopic Particle count and Analysis}

Particles dispersed in the liquid (droplet) are transported by the flow and deposited or assembled on a solid surface as the solvent evaporates. Depending on solid-surface properties and particle concentration, particles may form monolayers, multilayers, or both. In the latter case, for instance, a monolayer segment might be formed at the center while a progressive accumulation of particles leads to the formation of cluster-ring-shaped patterns after drying is complete, which often results in multilayers $[35,36]$. In the present study, particles were deposited and adsorbed on a hydrophilic $\mathrm{Si}$ surface. Generally, such a surface is highly adhesive and allows convective flow of fluid carrier thereby leading to the self-assembly of particles upon solvent evaporation.

Initially, MPS particle count estimate (from SEM micrographs) for single droplets deposited on Si bulk substrates was found to be 104,470 \pm 420 . However, by tuning the particle concentration and droplet size, a small particle assembly was realized on the cantilever surface (as shown in Figure 11). Prior to SEM particle-imaging, the resonant frequency response due to the attached particles (on the cantilever) was measured and the corresponding mass was determined thereof. The same sample was then mounted in the SEM and particle-imaging was performed. Subsequently, the number of particles and adsorbate position $(x)$ were estimated in ImageJ [24].

(a)
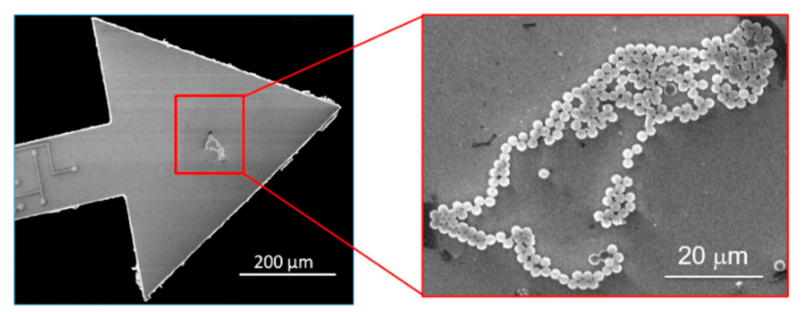

(b)
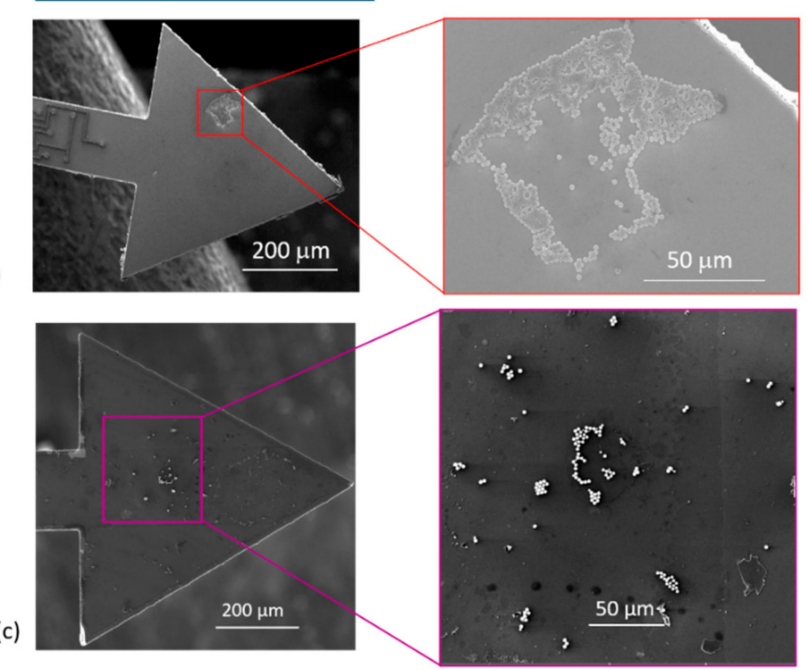

(d)
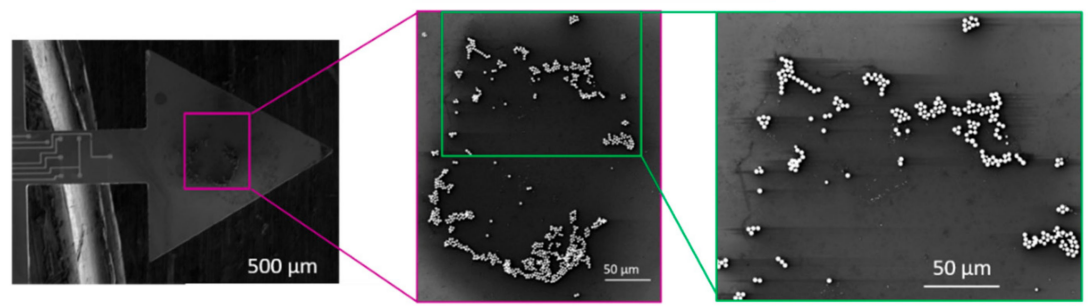

Figure 11. SEM images of adsorbed particles on cantilevers depicting monolayer particle distribution profiles: (a,b) sensors consisting of adsorbed MPS particles; and (c,d) sensors adsorbed with PMMA particles. Monolayer particles in (c,d) are positioned along the axisymmetric axis, whereas in (a) the particles were adsorbed nearly at center of the equilateral triangular surface. In (b), however, some MPS particles were adsorbed very close to the edge of the cantilever. 
In every measurement cycle, the cantilever was cleaned as described above. Typically, a monolayer particles distribution of the adsorbed on the sensors was observed (Figure 11). For MPS, SEM particle-counts of about $19 \pm 2$ (Figure $8 c$, inset), $60 \pm 6$ (Figure 8d, inset) and $203 \pm 8$ (Figure 11a) were determined, whereas, for PMMA, the counted particles were $47 \pm 6$ (least count), $123 \pm 8$ (Figure 11c) to $671 \pm 36$ (Figure 11d). The particle agglomeration patterns for both MPS and PMMA (as depicted in the SEM images shown in Figure 11) evidently show a similar trend. In some other instances, however, a monolayer plus cluster(s) of non-uniform multilayers was observed (Figure 12). In such a case, only particles on the top layer could be counted with certainty to give a minimum number of the adsorbate (e.g., >300).

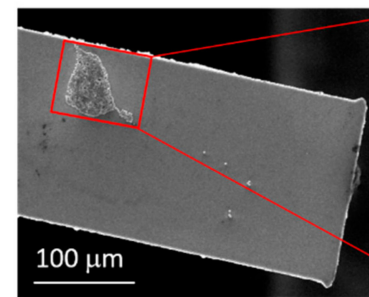

(a)

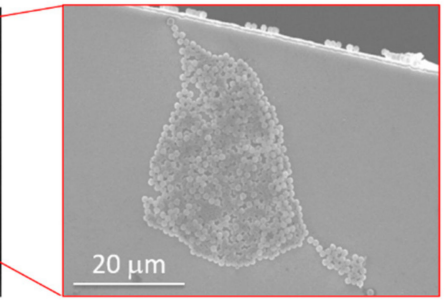

(b)

Figure 12. SEM images of MPS particles deposited on a rectangular cantilever (a); and the magnified view depicting a multilayered particles arrangement $(\mathbf{b})$.

In Figure 13, we further present a summary of MPS particle counts (from SEM imaging) and compare the same with the number of particles calculated from resonant frequency measurements. For the same adsorbate, the number of particles due to both point-mass (Equation (4)) and distributed-mass $\left(\Delta m=-2 m_{\mathrm{eff}} \Delta f / f_{0}\right)$ conditions was calculated. From both mass distributions, some disparities in the number of particles were observed. Nonetheless, the number of particles due to point mass was fairly comparable with the particles-count results from the SEM. Generally, the observed disparities in the number of particle (from the two approaches) were attributed to: some drift and inconsistencies in positioning of the dispensing tip (and therefore the droplet on the sensor), and formation of multilayers. The former is a consequence of tip misalignment and may lead to spilling or adsorbing of particles along the edges of the beam (as depicted in Figure 12b), whereas the latter introduced uncertainties in particle-count estimations.

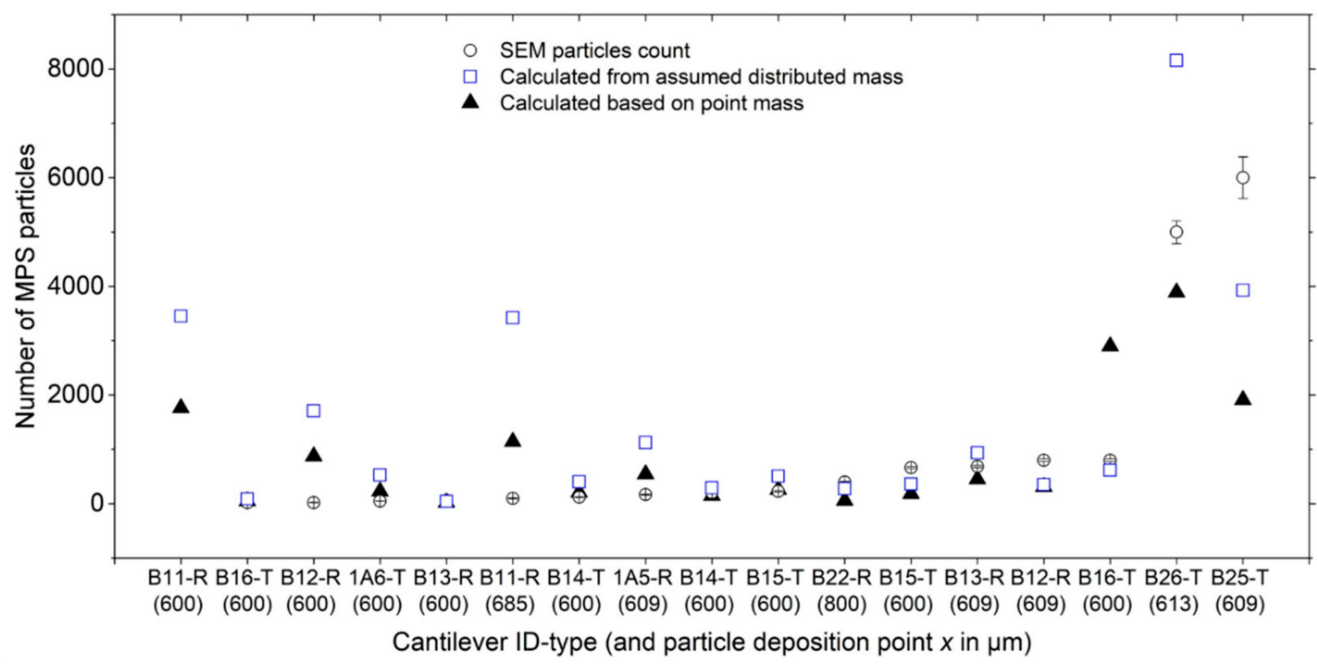

Figure 13. Comparison of the number of MPS particles calculated from resonant-frequency shifts (considering point-mass and distributed-mass conditions) with the particle-count results from SEM imaging. The last letter in the cantilever ID-type, i.e., $\mathrm{T}$ and $\mathrm{R}$, denote triangular and rectangular cantilever types, respectively. 


\subsection{Accuracy of Mass Measurements}

In sensing and determining the mass due to frequency shift induced by adsorbed particles or otherwise, knowledge of the measurement environment (especially relative humidity $\mathrm{r} H$ and temperature $T$ ) plays a critical role. In our study, these parameters ( $\mathrm{rH}$ and $\mathrm{T}$ ) were adequately monitored in every measurement cycle and found to be relatively stable. It is however worth noting that some little water from ambient atmosphere would possibly be adsorbed on the sensing surface courtesy of the hydrophilicity of our sensing surface and magnetic particles. In the current study, because of stable $\mathrm{rH}$ environment, we considered its contribution to the calculated mass to be negligible.

The position of particles on the sensing surface also affects the frequency response of the sensor due to the added mass (Equation (4)). Depositing particles away from the intended axisymmetric or eccentric target points would therefore clearly influence such output. In our work, such observations were evidently shown (e.g., Figures 11 b and 12b) and were mainly associated with the misalignment of the dispensing tip and/or relative change of the wettability of the sensing surface. The latter is possibly due to the interplay of wet cleaning(s) of the sensors and natural oxidation of sensing surface, parameters which can potentially tune the already hydrophilic sensing surface. If this surface is rendered more hydrophilic, the droplet may rapidly spread on the sensing surface and overflow unto the edges. Contrarily, spreading of liquid surface on less hydrophilic surface is slow and the droplet (hence, particles) can be fairly localized. Further work would therefore be necessary to enhance positioning with a better uncertainty. Amongst such considerations include coating the edges of the cantilever with a hydrophobic substance such as trimethoxy (octadecyl) silane (OTMS). This chemical compound would substantially help in confining the droplet within the sensing area and, hence, eliminate or minimize possible spillages. Basically, OTMS is compatible and forms strong covalent bonds with silicon oxide surfaces [37]. For our microcantilevers, such coating procedure would be integrated and performed in the last stages of the fabrication process.

Moreover, to enhance mass sensitivity towards detectability of single liquid-borne particles, scaling down of our micro-sized sensors is further intended. In our recent works, efforts towards this course have successfully culminated to a self-readable resonant cantilever of a mass of $m_{0}=2-5 \mathrm{ng}$ and a sensitivity of about $7.8 \mathrm{fg} / \mathrm{Hz}$ meant for the detection of airborne nanoparticles [38,39]. Nonetheless, in Figure 14, we illustrate our intended integration of tipless cantilever chips (PRSA-L300-F80-TL, from SCL-Sensor. Tech. Fabrication GmbH, https://www.sclsensortech.com/self-sensing-cantilevers-tipless/) with $m_{0} \sim 0.5 \mu \mathrm{g}$ (a factor of 30 and 50 lower than our rectangular and triangular cantilevers, respectively) into our measurement setup. These sensors offer the possibility of sensing micro particles with increased sensitivity. Calibration of these cantilever mass sensors would also favorably be considered to give minimal bias between the resonant measurement and SEM-particle count estimations.

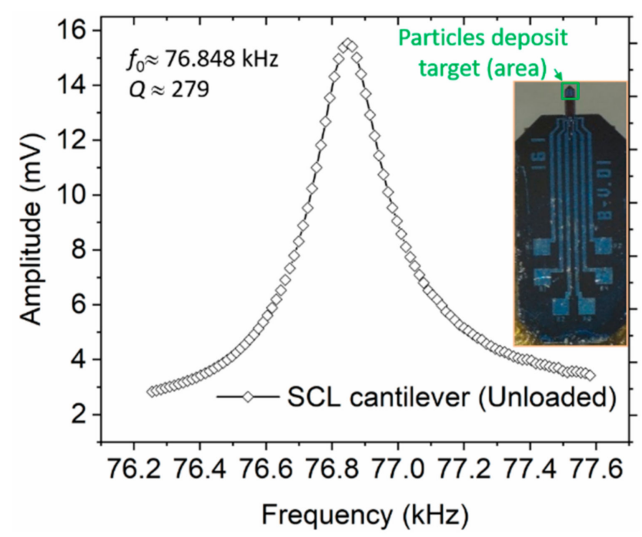

Figure 14. Measured frequency of a SCL tipless cantilever sensor. Inset is the photo of the sensor illustrating the possible particle-adsorption and -sensing area. 


\section{Conclusions}

In this work, we sampled and adsorbed liquid dispersed particles on our in-house fabricated silicon-based piezoresistive cantilever sensors and further successfully tested the same approach on $\mathrm{CiS}$ commercial cantilever sensors. The mass sensitivities of our sensors ranged from about $27 \mathrm{~Hz} / \mathrm{ng}$ to $14.73 \mathrm{~Hz} / \mathrm{ng}$ for rectangular and triangular free-ended cantilever, respectively. With these cantilever sensors, we determined the masses of attached water droplets and particles through resonant frequency responses and compared the findings thereof with SEM particle count analysis. In our measurement process, droplet dispensing parameters were optimized to realize a small water droplet volume $V_{\mathrm{d}}=49.7 \pm 1.9 \mathrm{pL}$ which perfectly fitted on the sensing surface under consideration. Furthermore, water droplet evaporation trends were also investigated to ascertain minimum waiting time prior to particle-mass measurements. In general, a total evaporation time $t_{\mathrm{ev}} \approx 39 \mathrm{~s}$ was determined and found to compare well with the theoretical estimation (37.14 s). Particle adsorption on hydrophilic Si bulk substrates and piezoresistive microcantilevers is also presented, followed by a determination of their mass and number based on both resonant frequency shift measurements and SEM image analysis for particle counting. Monolayer particles assembly on the sensor with a lowest particle count of about 19 and 47 for MPS and PMMA particles were, respectively, realized with a high-quality factor $Q \approx 1900$, which nearly corresponds to $Q$ of a bare cantilever.

Author Contributions: Conceptualization and methodology, W.O.N.; validation, W.O.N., A.S., H.B. and E.P.; formal analysis, W.O.N.; investigation, W.O.N. and A.S.; resources, H.B. and E.P.; writing-original draft preparation, W.O.N.; writing-review and editing, W.O.N., A.S., M.B., H.B. and E.P.; supervision, H.B. and E.P.; project administration, E.P.; and funding acquisition, E.P.

Funding: This project has received funding from the EMPIR program co-financed by the Participating States and from the European Union's Horizon 2020 research and innovation program under No. 17IND05 MicroProbes.

Acknowledgments: W.O.N. is grateful to the German Federal Ministry for Economic Cooperation and Development (BMZ) for a doctoral scholarship within the Braunschweig International Graduate School of Metrology (B-IGSM). A.S. acknowledges support from the Ministry of Research, Technology and Higher Education of the Republic of Indonesia under No. 37/RISET-Pro/FGS/III/2019 (World Bank Loan No. 8245-ID). M.B. is grateful for the support received from Niedersächsisches Vorab, Germany, through the "Quantum- and Nanometrology (QUANOMET)" initiative within the project NP 2-2. The technical support given by Angelika Schmidt, Juliane Breitfelder, Aileen Michalski, Andreas Heidemann and Karl-Heinz Lachmund is also gratefully acknowledged. We thank Alexander Deutschinger, SCL-Sensor. Tech. Fabrication GmbH, for providing the PRSA-L300-F50-TL cantilever.

Conflicts of Interest: The authors declare no conflict of interest.

\section{References}

1. Datar, R.; Kim, S.; Jeon, S.; Hesketh, P.; Manalis, S.; Boisen, A.; Thundat, T. Cantilever Sensors: Nanomechanical Tools for Diagnostics. MRS Bull. 2009, 34, 449-454. [CrossRef]

2. Boisen, A.; Dohn, S.; Keller, S.S.; Schmid, S.; Tenje, M. Cantilever-like micromechanical sensors. J. Micromech. Microeng. 2011, 74, 36101. [CrossRef]

3. Senesac, L.; Thundat, T.G. Nanosensors for trace explosive detection. Mater. Today 2008, 11, 28-36. [CrossRef]

4. Setiono, A.; Xu, J.; Fahrbach, M.; Bertke, M.; Nyang'au, W.O.; Wasisto, H.S.; Peiner, E. Real-Time Frequency Tracking of an Electro-Thermal Piezoresistive Cantilever Resonator with ZnO Nanorods for Chemical Sensing. Chemosensors 2019, 7, 2. [CrossRef]

5. Hawe, A.; Zölls, S.; Freitag, A.; Carpenter, J.F. Subvisible and Visible Particle Analysis in Biopharmaceutical Research and Development. In Biophysical Characterization of Proteins in Developing Biopharmaceuticals; Elsevier: Amsterdam, The Netherlands, 2015; pp. 261-286, ISBN 9780444595737.

6. Xu, S.; Mutharasan, R. Cantilever biosensors in drug discovery. Expert Opin. Drug Discov. 2009, 4, 1237-1251. [CrossRef]

7. Yoon, T.J.; Kim, J.S.; Kim, B.G.; Yu, K.N.; Cho, M.H.; Lee, J.K. Multifunctional nanoparticles possessing a "magnetic motor effect" for drug or gene delivery. Angew. Chem. 2005, 44, 1068-1071. [CrossRef] 
8. Wang, W.; Luo, J.; Wang, S. Recent Progress in Isolation and Detection of Extracellular Vesicles for Cancer Diagnostics. In Advanced Healthcare Materials; WILEY-VCH Verlag GmbH \& Co. KGaA: Weinheim, Germany, 2018; Volume 7, p. e1800484. [CrossRef]

9. Malvar, O.; Ruz, J.J.; Kosaka, P.M.; Domínguez, C.M.; Gil-Santos, E.; Calleja, M.; Tamayo, J. Mass and stiffness spectrometry of nanoparticles and whole intact bacteria by multimode nanomechanical resonators. Nat. Commun. 2016, 7, 13452. [CrossRef]

10. Burg, T.P.; Godin, M.; Knudsen, S.M.; Shen, W.; Carlson, G.; Foster, J.S.; Babcock, K.; Manalis, S.R. Weighing of biomolecules, single cells and single nanoparticles in fluid. Nature 2007, 446, 1066-1069. [CrossRef]

11. Alassi, A.; Benammar, M.; Brett, D. Quartz Crystal Microbalance Electronic Interfacing Systems: A Review. Sensors 2017, 17, 2799. [CrossRef]

12. Kapuscinski, M.; Agthe, M.; Bergström, L. Time-resolved viscoelastic properties of self-assembling iron oxide nanocube superlattices probed by quartz crystal microbalance with dissipation monitoring. J. Colloid Interface Sci. 2018, 522, 104-110. [CrossRef]

13. Park, M.; Choi, H.; Kim, S.J.; Kim, C.S.; Kouh, T. Resonance Frequency Shift of Microcantilever via Surface Adsorption of Magnetic Nanoparticles. J. Korean Magn. Soc. 2018, 28, 49-52. [CrossRef]

14. Ruffert, C. Magnetic Bead-Magic Bullet. Micromachines 2016, 7. [CrossRef] [PubMed]

15. Williams, H.M. The application of magnetic nanoparticles in the treatment and monitoring of cancer and infectious diseases. Biosci. Horiz. Int. J. Stud. Res. 2017, 10, 257. [CrossRef]

16. Gul, S.; Khan, S.B.; Rehman, I.U.; Khan, M.A.; Khan, M.I. A Comprehensive Review of Magnetic Nanomaterials Modern Day Theranostics. Front. Mater. 2019, 6, 928. [CrossRef]

17. Akbarzadeh, A.; Samiei, M.; Davaran, S. Magnetic nanoparticles: Preparation, physical properties, and applications in biomedicine. Nanoscale Res. Lett. 2012, 7, 144. [CrossRef] [PubMed]

18. Stark, W.J.; Stoessel, P.R.; Wohlleben, W.; Hafner, A. Industrial applications of nanoparticles. Chem. Soc. Rev. 2015, 44, 5793-5805. [CrossRef] [PubMed]

19. Mehdizadeh Taheri, S.; Michaelis, M.; Friedrich, T.; Förster, B.; Drechsler, M.; Römer, F.M.; Bösecke, P.; Narayanan, T.; Weber, B.; Rehberg, I.; et al. Self-assembly of smallest magnetic particles. Proc. Natl. Acad. Sci. USA 2015, 112, 14484-14489. [CrossRef]

20. Gubin, S.P.; Spichkin, Y.I.; Yurkov, G.Y.; Tishin, A.M. Nanomaterial for High-Density Magnetic Data Storage. Russ. J. Inorg. Chem. 2002, 47, 32-67.

21. Mohammed, L.; Gomaa, H.G.; Ragab, D.; Zhu, J. Magnetic nanoparticles for environmental and biomedical applications: A review. Particuology 2017, 30,1-14. [CrossRef]

22. Egan, J.G.; Hynes, A.J.; Fruehwald, H.M.; Ebralidze, I.I.; King, S.D.; Alipour Moghadam Esfahani, R.; Naumkin, F.Y.; Easton, E.B.; Zenkina, O.V. A novel material for the detection and removal of mercury(ii) based on a 2,6-bis(2-thienyl)pyridine receptor. J. Mater. Chem. C 2019, 7, 10187-10195. [CrossRef]

23. Hamdana, G.; Südkamp, T.; Descoins, M.; Mangelinck, D.; Caccamo, L.; Bertke, M.; Wasisto, H.S.; Bracht, H.; Peiner, E. Towards fabrication of 3D isotopically modulated vertical silicon nanowires in selective areas by nanosphere lithography. Microelectron. Eng. 2017, 179, 74-82. [CrossRef]

24. Rueden, C.T.; Schindelin, J.; Hiner, M.C.; DeZonia, B.E.; Walter, A.E.; Arena, E.T.; Eliceiri, K.W. ImageJ2: ImageJ for the next generation of scientific image data. BMC Bioinform. 2017, 18, 529. [CrossRef] [PubMed]

25. Stalder, A.F.; Melchior, T.; Müller, M.; Sage, D.; Blu, T.; Unser, M. Low-bond axisymmetric drop shape analysis for surface tension and contact angle measurements of sessile drops. Colloids Surf. A Physicochem. Eng. Asp. 2010, 364, 72-81. [CrossRef]

26. Alam, A.U.; Howlader, M.M.R.; Deen, M.J. The effects of oxygen plasma and humidity on surface roughness, water contact angle and hardness of silicon, silicon dioxide and glass. J. Micromech. Microeng. 2014, 24, 35010. [CrossRef]

27. Wasisto, H.S.; Merzsch, S.; Uhde, E.; Waag, A.; Peiner, E. Handheld personal airborne nanoparticle detector based on microelectromechanical silicon resonant cantilever. Microelectron. Eng. 2015, 145, 96-103. [CrossRef]

28. Bertke, M.; Hamdana, G.; Wu, W.; Wasisto, H.S.; Uhde, E.; Peiner, E. Analysis of asymmetric resonance response of thermally excited silicon micro-cantilevers for mass-sensitive nanoparticle detection. J. Micromech. Microeng. 2017, 27, 64001. [CrossRef]

29. Schmid, S.; Villanueva, L.G.; Roukes, M.L. Fundamentals of Nanomechanical Resonators; Springer International Publishing: Cham, Switzerland, 2016; ISBN 978-3-319-28689-1. 
30. Dohn, S.; Svendsen, W.; Boisen, A.; Hansen, O. Mass and position determination of attached particles on cantilever based mass sensors. Rev. Sci. Instrum. 2007, 78, 103303. [CrossRef]

31. Toledo, J.; Ruiz-Díez, V.; Bertke, M.; Suryo Wasisto, H.; Peiner, E.; Sánchez-Rojas, J.L. Piezoelectric MEMS Resonators for Cigarette Particle Detection. Micromachines 2019, 10, 145. [CrossRef]

32. Arcamone, J.; Dujardin, E.; Rius, G.; Pérez-Murano, F.; Ondarçuhu, T. Evaporation of femtoliter sessile droplets monitored with nanomechanical mass sensors. J. Phys. Chem. B 2007, 111, 13020-13027. [CrossRef]

33. Erbil, H.Y.; McHale, G.; Newton, M.I. Drop Evaporation on Solid Surfaces: Constant Contact Angle Mode. Langmuir 2002, 18, 2636-2641. [CrossRef]

34. Cussler, E.L. Diffusion. Mass Transfer in Fluid Systems, 3rd ed.; Cambridge University Press: Cambridge, NY, USA, 2009; ISBN 9780511478925.

35. Nyang'au, W.O.; Setiono, A.; Puranto, P.; Bertke, M.; Wasisto, H.S.; Viereck, T.; Bosse, H.; Peiner, E. Droplet-on-cantilever approach for determining the mass of magnetic particles. In Proceedings of the 20. GMA/ITG-Fachtagung Sensoren und Messsysteme 2019, Nürnberg, Germany, 25-26 June 2019; AMA Service GmbH: Wunstorf, Germany, 2019; pp. 222-229. [CrossRef]

36. Anyfantakis, M.; Baigl, D. Manipulating the Coffee-Ring Effect: Interactions at Work. Chemphyschem 2015, 16, 2726-2734. [CrossRef] [PubMed]

37. Ressier, L.; Viallet, B.; Grisolia, J.; Peyrade, J.P. Chemical patterns of octadecyltrimethoxysilane monolayers for the selective deposition of nanoparticles on silicon substrate. Ultramicroscopy 2007, 107, 980-984. [CrossRef] [PubMed]

38. Bertke, M.; Xu, J.; Fahrbach, M.; Setiono, A.; Wasisto, H.S.; Peiner, E. Strategy toward Miniaturized, Self-out-Readable Resonant Cantilever and Integrated Electrostatic Microchannel Separator for Highly Sensitive Airborne Nanoparticle Detection. Sensors 2019, 19, 901. [CrossRef] [PubMed]

39. Bertke, M.; Xu, J.; Setiono, A.; Kirsch, I.; Uhde, E.; Peiner, E. Fabrication of a micro-cantilever-based aerosol detector with integrated electrostatic on-chip ultrafine particle separation and collection. J. Micromech. Microeng. in press (accepted).

(C) 2019 by the authors. Licensee MDPI, Basel, Switzerland. This article is an open access article distributed under the terms and conditions of the Creative Commons Attribution (CC BY) license (http://creativecommons.org/licenses/by/4.0/). 